\title{
Application of sliding mode in the construction of reinforced concrete Hejun $\mathrm{Xu}^{1}$, Yuyan Huang ${ }^{2}$, Chao Jiao ${ }^{1} \mathrm{Yu} \mathrm{Wang}^{3}{ }^{3}$ \\ ${ }^{1}$.The School of Civil Engineering, Shenyang Jianzhu University, Liaoning Shenyang 110168 \\ 2.The School of material engineering, Shenyang Jianzhu University, Liaoning Shenyang 110168 \\ ${ }^{3}$ The School of Civil Engineering, staff room of engineering mechanics, ShenyangJianzhu University, Liaoning Shenyang 110168
}

Keyword: RC slidingmode; Construction; RC silo

\begin{abstract}
The sliding formwork construction is a new technology of cast-in-place concrete project, com-pared with the conventional construction method, synovial fast construction speed, high degree of mechanization, can save formwork and scaffold required quantity, can be more convenient and flexible template apart will be assembled and can be reused. The article for a new kind of reinforced concrete structure, form of syn-ovial membrane were discussed in detail, and has carried on the analysis to its application in construction.
\end{abstract}

\section{Sliding mode structure described}

\subsection{The technical background}

The cast-in-place concrete structure engineering for construction of building template structure, mainly composed of a panel, the supporting structure and the connecting piece is composed of three parts. The panel is in direct contact with the bearing plate of fresh concrete; support structure is the temporary structure support panel, concrete andconstruction load, guarantee the structure building templates are firmly combined, do not deformation, not destroy; the connecting piece is connected into the panel and the supporting structure parts of the whole. ${ }^{[1]}$

The fixed template in building construction, not only cost is high, and the disassembly and assembly are the need of professionals, and spend a lot of manpower and resources,increase the cost of the project, to extend the time limit for a project, is not conducive to the development of modern construction industry.

\subsection{The content and structure}

The technical problem of reinforced concrete sliding mode to solve, is to address the above problems of technology, provides a special reinforced concrete sliding mode, a baffle andenclosure fixed form templates, Jack in jack frame along the rod climbing movement, thereby driving the construction template rises, cancel the fixed template, fixed template for slip type movable steel die, so do not need to prepare a large number of fixed template erection. ${ }^{[2]}$

In order to solve the technical problem, the technical scheme adopted is: synovial includingjack, jack frame, pole climbing and template, two horizontal fixed jack frame on both sides are respectively provided with two templates, and the template plane perpendicular to the jackframe is fixed; Jack is fixed on the joists center position, stringer is horizontally arranged in thejacks frame; the pole climbing vertical penetrating beam jack frame, and pole climbing through the stringers and jack fixed; two jacks and a high pressure oil pipe in series, the other end of the high pressure oil pipe connected with the fuel line of the high-pressure fuel pump; the lower end part of the climbing rod is fixedly provided with a supporting plate, a supporting plate in the length direction and jack plane vertical. Jack comprises a lifting column and cylinder, cylinder and pole climbing through after lifting column fixation. The template includesenclosure and a baffle, the baffle is installed on the upper and lower ends outside theenclosure, and the baffle plate and the enclosure is vertical to the length direction.

The baffle plate and the enclosure are connected through a stud and nut, the baffle plate are arranged on the inner side of the nut hole.

Compared with the prior art, the synovial membrane has the following advantages: 1, Jack injack 
frame along the rod climbing movement, so as to enhance the template increases in height, has changed the traditional fixed template, inefficient construction need to re disassembly can re construction; 2, high-pressure tubing and two jack oil road series,ensuring the work when the template is also rising, to prevent the occurrence of the phenomenon of high-low plate, speed up the construction progress; 3, the baffle plate are arranged on the inner side of the nut in the hole position, the nut just into the nut holes,reduces the friction and avoid the emergence of baffle baffle concave convex surface. ${ }^{[3]}$

\subsection{Sliding mode structure design}
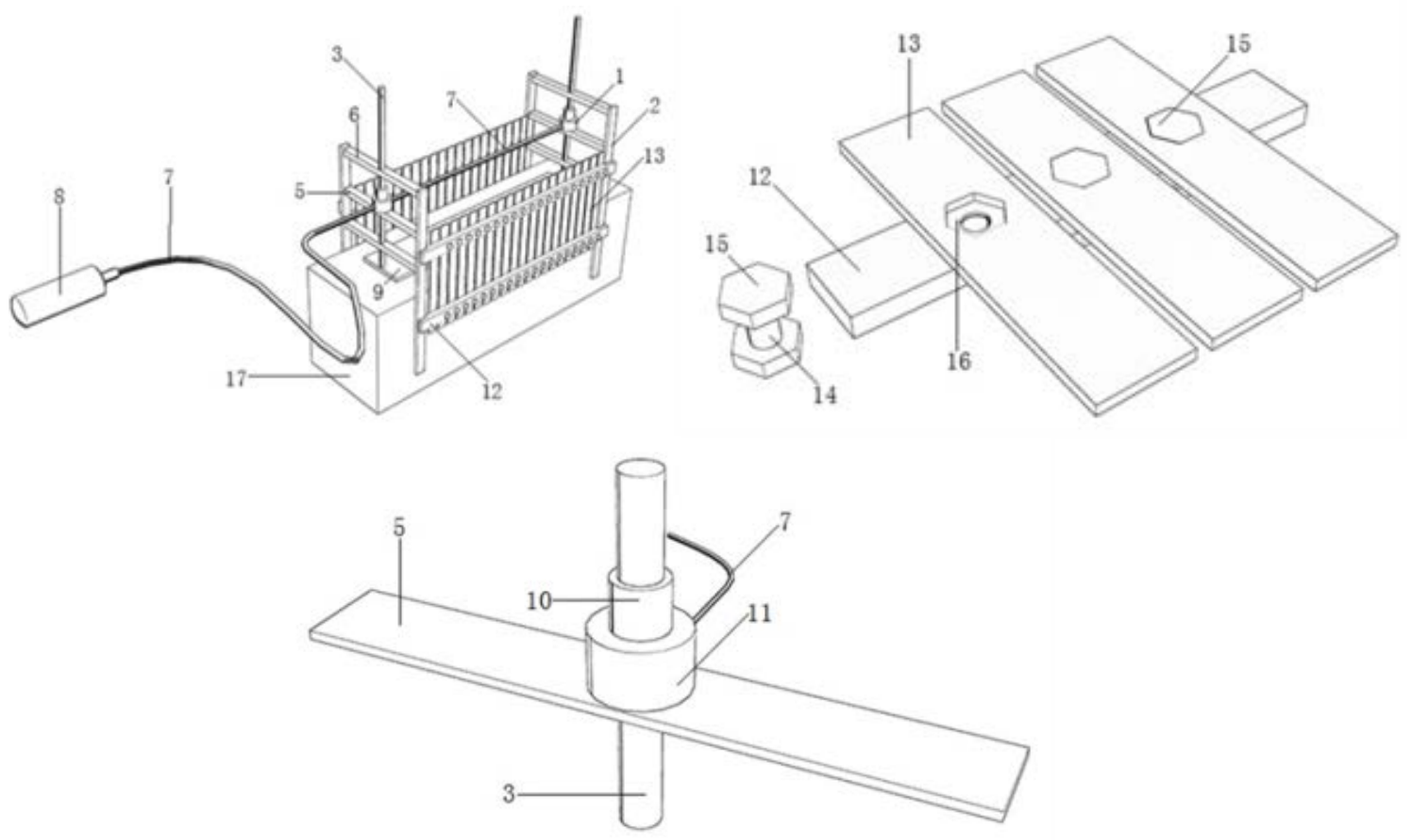

\section{Application method in construction}

\subsection{The construction scheme of the multi-storey residential building}

Combined with last picture, including a jack, jack frame 2, 3 and templates climbing pole 4, two horizontal fixed jack stand two sides were fitted with two templates 4 and 4 template plane perpendicular to the jack frame 2 fixed; jack a fixed position in the beam centered on five, five horizontal beams mounted in the jack stand 2; 3 vertical pole climbing frame beams 2 through 6 jacks and climbing pole 3 through 5 and the jack a stringer fixed; two jack 1 in series with a high-pressure tubing 7, high-pressure tubing and the other end with the high pressure pump 8 is connected to an oil passage 7; the lower end portion of the rod 3 is fixed climbing support plate 9 is mounted, support plate 9 and the jack frame 2 in the plane perpendicular to the longitudinal direction ; jack 1 includes lifting column 10 and cylinder 11, after climbing pole 3 through 11 with the lifting cylinder 10 is fixed to the column; 4 includes templates baffle enclosure 12 and 13, the upper and lower ends of the outer flaps 13 are mounted enclosure 12, and baffle enclosure 13 and 12 in the longitudinal direction perpendicular; baffle 131215 connected with the enclosure through the studs and nuts 14, 13 have a nut inside of the bezel opening 16 holes.

High-pressure pump 8 through the high pressure hydraulic hose 7 to jack a job, a jack lifting column on the drive lever 3 climb downward movement of the cylinder 11 due to the stringers 5 fixed cylinder 11 to drive along the climbing frame 2 jack up rod 3 sports, installation will reach 9 supporting role in the bottom of the climbing pole three gussets in the cement wall 17, thereby ensuring a template 4 remains horizontal, template 4 by baffles 13 and 12 around the circle with 14 studs and nuts 15 composed of the baffle 13 holes opened a nut 16, reducing the template 4 and 17 cement 
wall friction, and avoid leaving marks on the cement wall bump 17, studs 14 and nuts 15 with an increase of 4 mounting template convenience and efficiency; jack on jack stand 123 movement along the climbing pole, which led to the rise of construction template 4 , the abolition of the fixed template, change the fixed template for skid active Template and thus do not need to prepare a large number of fixed template set up, reduced the duration and reduce the construction cost, efficient and convenient, more suitable for the construction of modern construction projects.

\subsection{The construction scheme of silo slip form cement factory}

The first part: steel banding monitoring:

First formulated reinforcement.Vertical steel silo given by $5 \mathrm{~m}$ long preparation, $1 / 4$ misconnection consideration. At the same time the level of the ring to the silo through a long length of reinforced steel bars to take a long, less than some change for pay laps. Located between the inner and outer double reinforced according to the construction requirements of small retractors.

Sliding steel silo which uses banding joints, vertical steel construction requirements lap by lap length, connector locations staggered arrangement, any horizontal section, section steel joints $1 / 4$ miswired; horizontal hoop reinforcement lap length $61 \mathrm{~d}$, joint position staggered arrangement allows every three steel set a lap joint in the same vertical section, inside and outside the level of reinforcement uniform, staggered.

After the vertical steel for position control. With 16 or 18 rings welded rebar reinforcement to locate on the beam, while the positioning ring on which the welding ring. Vertical steel retaining ring to the socket to ensure that the size and spacing of the vertical thickness of the protective layer of steel. Where each vertical bars are through the slip ring center. Slip rings connected by location long vertical steel banding When tendons, ensure the lower end of the vertical ribs connected under the slip ring, avoid slip ring connectors hook. Ring bars construction requirements set by the skeleton ribs, bent pitch control banding lap length.

Part II: reserved mounting holes and embedded control:

Before sliding model, to set aside all the work embedded detailed statistics, listing forms, marked elevation, location, reservations embedded varieties, specifications, embedded in more complex place, do beforehand on Sliding platform good sign. Various embedded parts shall be reserved to the external ring bars welded, attention must be symmetrical on both sides of the reserved hole concrete pouring. According to the construction requirements, set aside the hole with boards nailed wooden embedded system, the outer wooden polishing, brushing release agent.

Part III: Concrete quality monitoring:

First, centralized site mixed concrete. Crane and lifting to sliding platform.

After pouring of concrete. Warehouse wall slip liters per car dumped in two lifting frame gap, the platform will be open hand shovel concrete into the mold, followed by vibrating vibrator workers. When continuous concrete pour right into the mold from a reverse and vibrators. Ensure the concrete top surface height of about $10 \mathrm{~cm}$ below the template. After the concrete into the mold promptly with plug-in vibrator vibrators, vibrators operation each time $10 \mathrm{~s} \sim 15 \mathrm{~s}$, stop vibrating when the concrete surface is no longer apparent sinking bubbles no longer appear when the surface of the pan syrup. Do leak vibration, excessive vibration, which do not comply with the provisions of the phenomenon.

According synovial climatic factors, Liaoning Province, the higher summer temperatures, to join the retarder in the concrete; colder in the winter, to take concrete measures to ensure the quality of the double synovial construction. Such as halogen lamp heating, double insulation measures.

Part IV: Control Sliding movements:

Template sliding across sectors need to work closely together, before sliding the preparatory work of various departments to ensure the safety, sliding platform system technically, security, must meet the quality requirements to ensure staff well-organized, timely supply of materials, water and electricity normal.

Rising phases: the first concrete pouring height appropriate for the $1000 \mathrm{~mm}$, the positive and negative points four pouring generally 4-6 hours began to test upgrade and enhance 3-4 trip. Strength, the initial template sliding to the ground floor pouring concrete strength of 0.15 
$0.35 \mathrm{Mpa} \mathrm{pm}$. When sliding test should be raised at the same time all the jack $8-10 \mathrm{~cm}$, fully observe the mold concrete strength, quality, the compliance with the requirements before the template slide rise $250-300 \mathrm{~mm}$ high. Then for all lifting equipment and a template system to conduct a comprehensive inspection. After trimming, can be transferred to the normal sliding, normal strength concrete mold release should be controlled at $0.15 \sim 0.35 \mathrm{Mpa}$.

After the initial slide, according to the normal coordination program plans pouring layered, layered sliding. Normal sliding, sliding time interval between the two, to be determined according to the concrete cube strength reached $0.15 \sim 0.35 \mathrm{MPa}$ time. Control of each layer pouring pouring height of 250-300mm, lashing one (pouring layers) reinforcement, pouring a layer of concrete, concrete positive and negative cycle to cast at higher temperatures enhance 1-2 midway trip, temperatures higher than then by lifting stroke is low. ${ }^{[4]}$

\section{Technical summary}

This technology uses jack, jack stands, climbing pole and templates, two horizontal sides of the fixed jack stand were installed with two templates, and template fixed plane perpendicular to the jack frame; jack fixed in the middle position on the stringers, stringers horizontally installed in the jack frame; climbing pole vertical beams through the jack stand and climb through the stringers and the jack rod fixed; two jacks and a high-pressure tubing series, high pressure tubing and the other end connected to the high-pressure pump oil passage; climb the lower end of the rod fixedly attached to support plate, support plate and jack frame perpendicular to the plane in the longitudinal direction.

Sliding baffle and enclosure using a fixed form a template, jack in the jack stand along the climbing pole movement, which led to the rise of construction template, the abolition of the fixed template, change the template for fixed steel skid activity. Combined with the construction of the building can be seen in two instances, the application of sliding mode in the construction, no need to prepare a large number of fixed template set up, reducing the amount of construction, speed up the construction speed.

\section{Acknowledgments}

The authors would like to thank associate professor Yu Wang for excellent technical support.He is the Corresponding author of this article.

\section{References}

[1]. Song Yupu "modern concrete basic theory and engineering application of" [M]. ChinaArchitectural Press 2008

[2]. Wang Qingxiang, Song Yupu "reinforced concrete structure" [M].2004 mechanical press

[3]. Wang Licheng, Wang Qingxiang, Song Yupu "reinforced concrete structure" [M].2008mechanical press

[4]. Kato atsuko,TanakaMinoru,Hiroshi Yoshino "new model influenza environment" [J].Model Building Environment Engineering Research Report 2012 annual Vol.4:23-27.

[5]. "reinforced concrete structure design code".[S] GB50010-2010

[6]. "reinforced concrete and prestressed concrete engineering specifications".[S] JTG - D62 -2004 